\title{
Crustal motion results derived from observations in the European geodetic VLBI network
}

\author{
Rüdiger Haas ${ }^{1}$, Erwan Gueguen ${ }^{2}$, Hans-Georg Scherneck ${ }^{1}$, Axel Nothnagel ${ }^{3}$, and James Campbell ${ }^{3}$ \\ ${ }^{1}$ Onsala Space Observatory (OSO), Chalmers University of Technology (CUT), SE-439 92 Onsala, Sweden \\ ${ }^{2}$ ITIS-CNR, Centro di Geodesia Spaziale, IT-75100 Matera, Italy \\ ${ }^{3}$ Geodätisches Institut der Universität Bonn (GIUB), Nußallee 17, DE-53115 Bonn, Germany
}

(Received December 22, 1999; Revised May 18, 2000; Accepted May 19, 2000)

\begin{abstract}
Geodetic VLBI observations have been performed with the European geodetic VLBI network since early 1990 on a regular basis. The purpose of these observations is to determine crustal motion in Europe and to establish a stable reference frame for other space geodetic techniques. Over the years the size of the network and the number of participating stations has steadily increased. Today, the network extends from the island of Sicily in the south to the island of Spitsbergen/Svalbard in the north and from the Iberian peninsula in the west to the Crimean peninsula in the east. The area covered by the network is affected by two main geodynamic processes which are post-glacial rebound effects in the northern part, and the evolution of the Alps-Apennines orogenic systems in the southern part. With nearly 10 years of VLBI observations the determination of crustal motion in Europe is carried out with high accuracy. Baseline measurements are achieved with an accuracy of a few parts per billion. We compare the evolution of baseline lengths and topocentric station displacements with geophysical models. Strain rates in Europe on a large scale are determined from the results of the VLBI analysis.
\end{abstract}

\section{Introduction}

In January 1990 the European geodetic Very Long Baseline Interferometry (VLBI) group, a community formed by members of institutions involved in carrying out geodetic VLBI observations joined their efforts to initiate a purely European geodetic VLBI programme on a regular basis. Between January 1990 and December 1999 a total number of 52 sessions has been carried out. Currently, six European geodetic VLBI sessions are being observed every year, in the year 2000 the number of sessions will be increased to eight per year.

The goals of the project are to determine crustal motion in Europe with geodetic VLBI and to provide a stable reference network for other geodetic techniques used in the area for densification purposes, e.g. regional Global Positioning System (GPS) networks.

The observing programme is coordinated by the Geodetic Institute of the University of Bonn (GIUB) and the VLBI data is correlated at the Mark-IIIA correlator of the Max-PlanckInstitute for Radioastronomy (MPIfR) in Bonn, Germany, by members of the GIUB.

Carrying out this multi-national research project requires extra operational costs for coordination, scheduling, shipping of magnetic tapes, data processing etc. that are beyond the regular budgets of the participating institutions. Therefore the European geodetic VLBI group applied for support by the European Union (EU). During the years 1993-1996 the project received grants from the EU within the 'Science' pro-

Copy right(C) The Society of Geomagnetism and Earth, Planetary and Space Sciences (SGEPSS); The Seismological Society of Japan; The Volcanological Society of Japan; The Geodetic Society of Japan; The Japanese Society for Planetary Sciences. gramme. Phase II of the project (1996-2000) is supported by the EU in the 'Training and Mobility of Researchers' (TMR) programme under the title 'Measurement of Vertical Motion in Europe by VLBI'. The grant includes five temporary post-doctoral visiting researcher positions in four different participating countries (Campbell, 1996).

\section{The European Geodetic VLBI Network}

Today the fixed-station European geodetic VLBI network consists of 10 stations: Onsala (Sweden), Wettzell (Germany), Madrid (Spain), Medicina (Italy), Matera (Italy), Noto (Italy), Effelsberg (Germany), Simeiz (Ukraine), Ny Ålesund (Norway) and Yebes (Spain).

The first purely European observations in 1990 were scheduled with the stations Onsala, Wettzell, Madrid, Medicina, Matera and Noto. In 1991 Effelsberg was equipped permanently with the necessary hardware which enables its participation in geodetic observations. From 1994 onwards the two stations Simeiz and Ny Ålesund provided the much needed extension of the geodetic network to the east and to the north. With the Yebes observatory a second station in Spain joined the network in 1995.

In the first two years of the project only three sessions per year were observed. In the following years six sessions per year were planned. Due to correlator restrictions only four could be observed in 1993. Because of the high demand for large telescopes from the astronomy community, the $100 \mathrm{~m}$ telescope of Effelsberg can only observe once or twice a year for geodesy. 


\section{The Geodynamic Setting}

The area covered by the network can be divided in a geodynamical sense into three main parts, the northern, the central and the southern part.

The northern part of the network, i.e. the Spitsbergen archipelago and Fennoscandia, had been covered by a substantial ice sheet in the Pleistocene. At present this area is under isostatic rebound after the vanishing of the ice shield about 10,000 years ago. Besides the obvious vertical movement horizontal deformation encompasses predominantly the periphery of the former ice shield, at the transition from the central dome to the fore-bulge area. This component of deformation is sensitive to lithosphere thickness and upper mantle viscosity. Maximal vertical uplifts in the centre of the previously glaciated area are predicted to be $12 \mathrm{~mm} / \mathrm{yr}$, maximal tangential deformation in the fore-bulge area is predicted to be $2 \mathrm{~mm} / \mathrm{yr}$ (Mitrovica et al., 1994). The VLBI stations Onsala and Ny Ålesund are both located in the post-glacial uplift area.

The central part of the network north of the Alpine system is regarded as the essentially 'stable' part of Europe although this part includes a zone of crustal weakness along the Rhine graben. The VLBI stations Wettzell and Effelsberg are located in this central part of the network south of the post-glacial fore-bulge area.

The southern part of the network, i.e. the western Mediterranean region, is dominated by the evolution of the Maghrebides-Appenines system and its related back-arc basin (Réhault et al., 1984; Dewey et al., 1989; Albarello et al., 1995; Gueguen et al., 1998) even if the driving mechanism is still controversial. According to Doglioni et al. (1997) and Gueguen et al. (1998) the subduction of the European margin under the Alps reached the collision stage and this led to the flip of the subduction zone, with the Appeninic system starting at the front of the back-thrust belt of the Alps. At the beginning the Apenninic subduction migrate eastward easily and quickly but around $10 \mathrm{Ma}$ ago the Apenninic front reached the thicker continental crust of the Apulia Platform and this has led to a drastic change of the geodynamics settings of the Apenninic system. In the southern Apennines the migration of the subduction hinge slowed down, whereas in the central and northern Apennines there still is a subduction of thin Adriatic lithosphere allowing roll-back of the hinge. Owing to these different migration rates the Apennines front was split in two "sub-arcs". Due to these changes in the rollback velocity, a precise determination of the motion of the Adriatic plate is fundamental to understand the present-day evolution of the Apennines system.

\section{Data Analysis}

The European geodetic VLBI sessions from 1990 to 1999 were analysed using the CALC/SOLVE/GLOBL VLBI data analysis software package (Ma et al., 1990). In our analysis relative clock parameters with respect to a reference clock in each session were estimated every 6 hours. In order to compensate for unmodelled atmospheric refraction effects, atmospheric zenith path delays were introduced as unknown parameters every 60 minutes using the NMF 2.0 mapping functions (Niell, 1996). Horizontal asymmetries in the atmospheric refraction profile were estimated with horizontal gradient parameters in north and east direction every 8 hours.

For our analysis we chose an approach which is usually called 'baseline-solution'. Each session was treated separately in a least-squares adjustment and station coordinates were estimated for all but one of the stations participating in the session. Since VLBI is a differential technique and the quasars observed are at effectively infinite distance from the earth, the coordinates of a reference station have to be fixed in the adjustment. In addition, the earth rotation parameters (pole coordinates and UT1) have to be introduced as a priori to permit the estimation of the station coordinates at all epochs. In our solution we adopted earth rotation parameters (ERP) together with radio source positions from a global VLBI solution by Ma and Ryan (1998). For more details on the different strategies of the analysis of VLBI data see Nothnagel (1993) and Nothnagel and Campbell (1993). Precession was modelled according to the IAU 1976 theory of precession (Lieske et al., 1977). Nutation offsets were estimated for each VLBI experiment with respect to the IAU 1980 theory of nutation (Seidelmann, 1982) which was used as a priori.

Refined models for the solid earth tides and ocean tide loading were applied instead of using the models recommended in the IERS Conventions (McCarthy, 1996). Solid earth tides were modelled as functions of frequency and latitude following Haas (1996) using the harmonic expansion of the tidal potential by Tamura (1987) and frequency and latitude dependent Love and Shida numbers by Wahr (1981) together with a Free Core Nutation $(\mathrm{FCN})$ period of 430 sidereal days. Ocean tide loading effects were introduced using a recent ocean tide loading model by Scherneck (1996) based on the CSR3.0 ocean tide model by the University of Texas (Eanes and Bettadpur, 1995), expanded by a large number of interpolated ocean tides. Atmospheric loading effects and thermal deformation of the VLBI telescopes was not yet accounted for in our analysis.

\section{Crustal Motion Results}

From the individual least-squares adjustments, time series of station coordinates become available which permit the computation of baseline components between individual stations for each epoch. The time series of baseline lengths and baseline components give insight into the repeatability of the measurements and the quality of the individual sessions. In addition, the components of motion in horizontal and vertical directions can also be inferred from the series of station coordinates. These topocentric station motions can then be used to perform strain rate analysis in a next step.

The advantage of baseline length results is that they are invariant to changes in the ERP. Thus, any precise ERP series is a sufficient basis if only baseline lengths are considered. The situation is slightly different if topocentric station coordinates and their evolution are derived from the time series of geocentric station coordinates. Here, the particular ERP series introduced will affect the orientation and the evolution of the network resulting in small rotations about the fixed reference point. The accuracy of the ERP series reported in Annual Reports of the International Earth Rotation Service (IERS) for the last 10 years is quoted to be on average 0.45 mas in all components. These uncertainties introduce 
additional noise in the topocentric station component time series. Test computations using this number have shown that, for example, the velocity components of Madrid which is separated from the fixed central point Wettzell by 1650 $\mathrm{km}$ are changed by up to $0.3 \mathrm{~mm} / \mathrm{y}$. The uncertainties of the other stations scale with their distance from Wettzell. Since these are worst case considerations it is safe to adopt ERP from the IERS or to use ERP from global VLBI analyses.

\subsection{Baseline length evolution}

In total there are 45 baselines in the network with baseline lengths from 99 to $4264 \mathrm{~km}$. Figure 1 shows the weighted root-mean-square errors (WRMS) of the regression lines for the baseline evolution versus baseline length. It is obvious that the repeatability of baseline measurements that include the station Simeiz is worse than for all other baseline measurements in the network. The station Simeiz has a rather short observation history and suffered from severe technical problems (Nesterov and Volvach, 1999). The repeatability of baseline measurements is as good as $2.1 \mathrm{~mm}$ plus an additional term depending on the length of the baseline of less than 1 part per billion (ppb), excluding the Simeiz baselines.

For the northern part of the network we compared $10 \mathrm{ob}-$ served baseline rates to predictions from a post-glacial rebound model. For the predictions we used the Ice-3G model (Tushingham and Peltier, 1991) together with the earth model by Mitrovica et al. (1994). The cross-correlation coefficient of predicted and observed baseline rates is 0.36 (see Fig. 2).

\subsection{Topocentric station movements}

In addition to the baseline oriented results we also produced station related horizontal and vertical drift components. The least-squares adjustment of the VLBI data set as described above yields a time series of station coordinates for all stations with respect to a reference station. In order to refer all results to a single reference station we excluded from this analysis three sessions in 1992 which did not involve Wettzell. From the individual station coordinates the

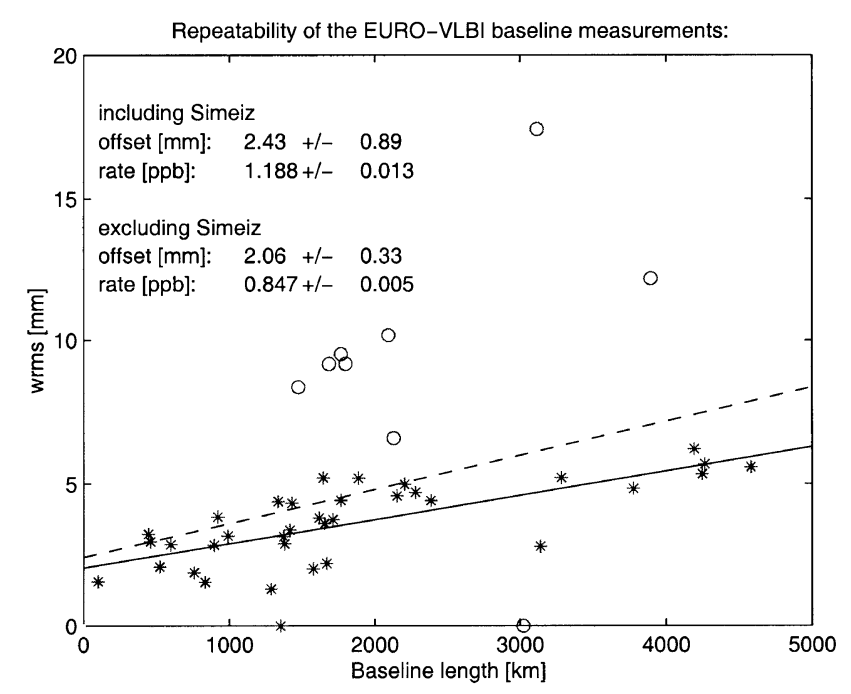

Fig. 1. Baseline repeatability in the European geodetic VLBI network. Baselines involving Simeiz are marked with circles, all other baselines are marked with asterisks. The regression line including all baselines is depicted as a dashed line, the one excluding baselines with Simeiz is drawn as a solid line.

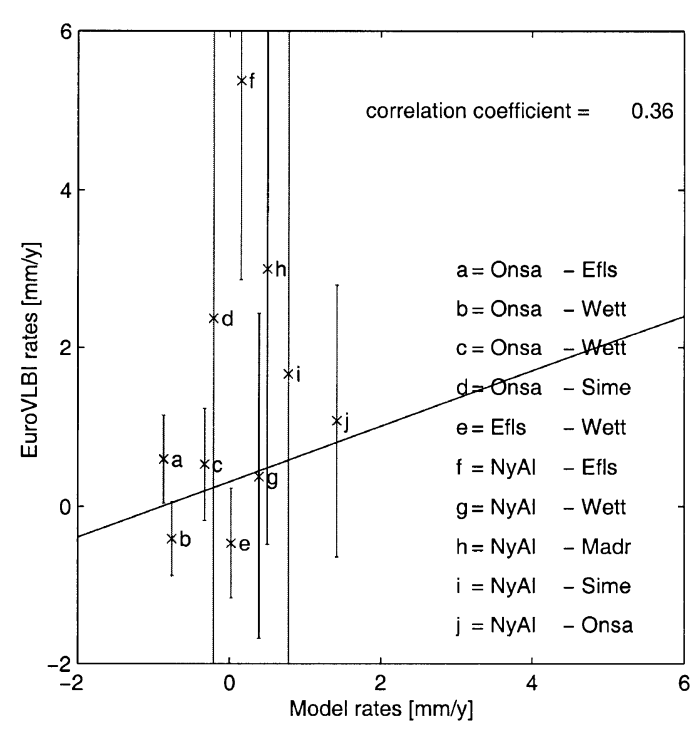

Fig. 2. Correlation plot of baseline rates of 10 baselines in the northern part of the European VLBI network. Shown are the rates derived from observations in the European VLBI network against model predictions, see text for details.

global drift of the Eurasian plate was subtracted according to the NUVEL-1A-NNR plate tectonic model (De Mets et al., 1994) producing a Europe-fixed system. After a transformation from Cartesian into ellipsoidal coordinates using the WGS84 ellipsoid parameters, the resulting time series yield the motion of each station in east, north and vertical direction. These time series were used to determine horizontal and vertical station velocities with respect to the Eurasian plate. At the three stations Medicina, Effelsberg and Madrid discontinuities in the station movement were introduced as unknowns at the times of track and wheel replacement. The site Yebes was not included because of its short observational history. Table 1 lists the results for horizontal and vertical velocities which are also shown in a graphic representation in Figs. 3 and 4 together with predictions of the post-glacial rebound model used earlier. The uncertainties given are the formal errors of the least-squares adjustment of station velocities from the time series of topocentric station positions. Latter were treated as correlated within each observing session but as uncorrelated between different observing sessions.

Fixing the motion of one site to a continental drift model such as NUVEL-1A-NNR and using high accuracy ERP from external sources places a reliable bound on the long term drift of the European continent as represented by the observing sites. Only the ERP have an effect on the determination of the drifts of the stations relative to Wettzell. The long term stability of Wettzell from global solutions appears extremely high (Ma and Ryan, 1998) resulting in Wettzell being a suitable reference site for our European analysis. Short term variations only increase the scatter of the results so that the drift vectors determined up to date deserve a high level of confidence.

For the stations Ny Ålesund and Onsala horizontal motions relative to Wettzell of $1.5 \pm 0.5 \mathrm{~mm} / \mathrm{yr}$ with azimuth of $274 \pm$ $30.6^{\circ}$ and $0.94 \pm 0.2 \mathrm{~mm} / \mathrm{yr}$ with azimuth of $238 \pm 12.2^{\circ}$, respectively, can be detected. Using the same post-glacial 
Table 1. Horizontal and vertical velocities in $[\mathrm{mm} / \mathrm{yr}]$ and wrms in $[\mathrm{mm}]$ observed in the European geodetic VLBI network.

\begin{tabular}{lcccccc}
\hline Station & east rate & wrms & north rate & wrms & vertical rate & wrms \\
\hline Effelsberg & $+0.3 \pm 0.3$ & 1.7 & $-0.8 \pm 0.3$ & 2.2 & $+1.6 \pm 3.3$ & 9.4 \\
Madrid & $+0.1 \pm 0.2$ & 2.3 & $-0.0 \pm 0.2$ & 1.9 & $+2.3 \pm 1.1$ & 12.5 \\
Matera & $+1.0 \pm 0.1$ & 1.5 & $+2.8 \pm 0.1$ & 2.1 & $+0.4 \pm 0.8$ & 12.2 \\
Medicina & $+0.9 \pm 0.1$ & 1.6 & $+1.7 \pm 0.1$ & 1.5 & $-4.8 \pm 1.1$ & 8.0 \\
Noto & $-0.3 \pm 0.2$ & 2.0 & $+3.1 \pm 0.2$ & 2.2 & $-0.5 \pm 1.0$ & 9.8 \\
Ny Ålesund & $-1.5 \pm 0.5$ & 2.2 & $+0.1 \pm 0.8$ & 5.7 & $+1.1 \pm 2.1$ & 11.4 \\
Onsala & $-0.8 \pm 0.2$ & 1.7 & $-0.5 \pm 0.2$ & 2.9 & $+2.3 \pm 0.9$ & 14.4 \\
Simeiz & $+0.6 \pm 0.9$ & 4.8 & $+0.5 \pm 0.9$ & 2.2 & $+0.9 \pm 5.1$ & 44.1 \\
\hline
\end{tabular}

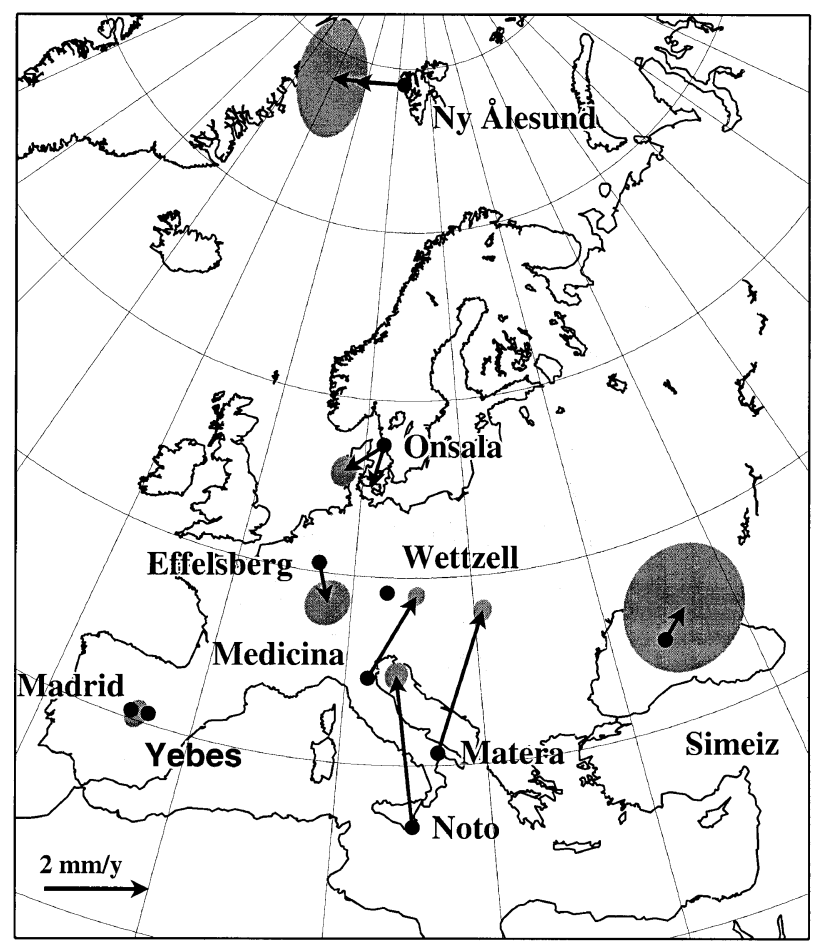

Fig. 3. Horizontal motions with respect to Wettzell: observations (vectors with error ellipses) and predictions due to a post-glacial rebound model (vectors without error ellipses).

rebound model as before, the predicted horizontal motions are $1.0 \mathrm{~mm} / \mathrm{yr}$ with azimuth $271.5^{\circ}$ for Ny ̊̊lesund and 0.9 $\mathrm{mm} / \mathrm{yr}$ with azimuth $193.9^{\circ}$ for Onsala, both with respect to Wettzell. The correspondence between measurements and predictions is rather good and for Onsala the difference in azimuth starts to get significant.

The horizontal movements of Effelsberg and Madrid with respect to Wettzell show that these parts of Europe currently do not experience any larger tectonic motion. The station Simeiz does not show significant horizontal motion since the measurement accuracy here is still not good enough.

In the Mediterranean area we detect the largest horizontal site motions in the European geodetic VLBI network. The Italian stations Medicina, Matera and Noto represent three

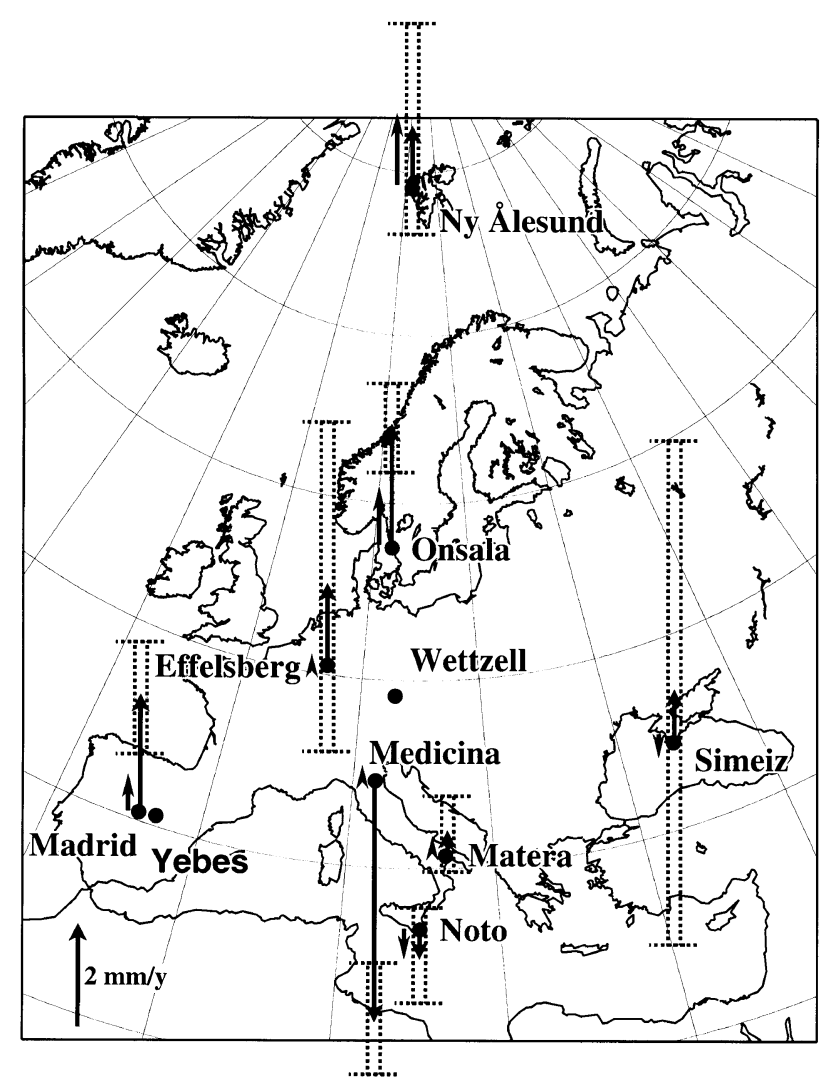

Fig. 4. Vertical motions with respect to Wettzell: observations (vectors with error bars) and predictions due to a post-glacial rebound model (vectors without error bars).

different geodynamic situations. Matera is the simplest one and clearly shows the displacement of the Adriatic plate. It shows a northward motion of $2.8 \mathrm{~mm} / \mathrm{yr}$ with respect to Wettzell and a slight eastward motion. The Medicina station is situated right on the front of the Apennines and so its situation is more complex to interpret. It shows a northward motion reduced to only $1.7 \mathrm{~mm} / \mathrm{yr}$ while the eastward motion is comparable to the one of Matera. The southernmost station Noto is in a completely different situation. Situated on the Hyblean microplate it shows evidence for being strongly affected by the African convergence. It moves northward by 
more than $3 \mathrm{~mm} / \mathrm{yr}$ with a light westward component with respect to Wettzell.

For the vertical station displacements we see uplifts at Ny Ålesund and Onsala of $1.1 \pm 2.1 \mathrm{~mm} / \mathrm{yr}$ and $2.3 \pm 0.9$ $\mathrm{mm} / \mathrm{yr}$, respectively, with respect to Wettzell. The value for Ny Ålesund still has a large error bar and cannot yet be considered as being really significant. Nevertheless post-glacial rebound models do predict an uplift for this station. Using again the same ice model and earth model as before, the predicted uplift rates relative to Wettzell are 1.3 and $1.0 \mathrm{~mm} / \mathrm{yr}$ at Ny Ålesund and Onsala, respectively, which corresponds reasonably well to the observed effects. The vertical motion results for Effelsberg and Simeiz are not significant yet.

The uplift at Madrid most probably does not have any geotectonic but rather a local explanation. An uplift of only about $0.5 \mathrm{~mm} / \mathrm{yr}$ can be explained by post-glacial rebound effects outside of the fore-bulge area. The Madrid GPS station operating for the International GPS Service (IGS) is separated about $275 \mathrm{~m}$ from the VLBI telescope and contributes data to the BIFROST project (Scherneck et al., 1998). Recent analysis shows for this GPS site an uplift rate of $4.5 \pm 0.4$ $\mathrm{mm} / \mathrm{yr}$. Conventional surveys raise suspicion that the causes for the uplifts seen with VLBI and GPS at this location have to be sought locally.

The detected subsidence at Medicina can be explained by extraction of ground water and gas in the Po valley (Tomasi et al., 1997). Matera and Noto do not show significant vertical site displacements.

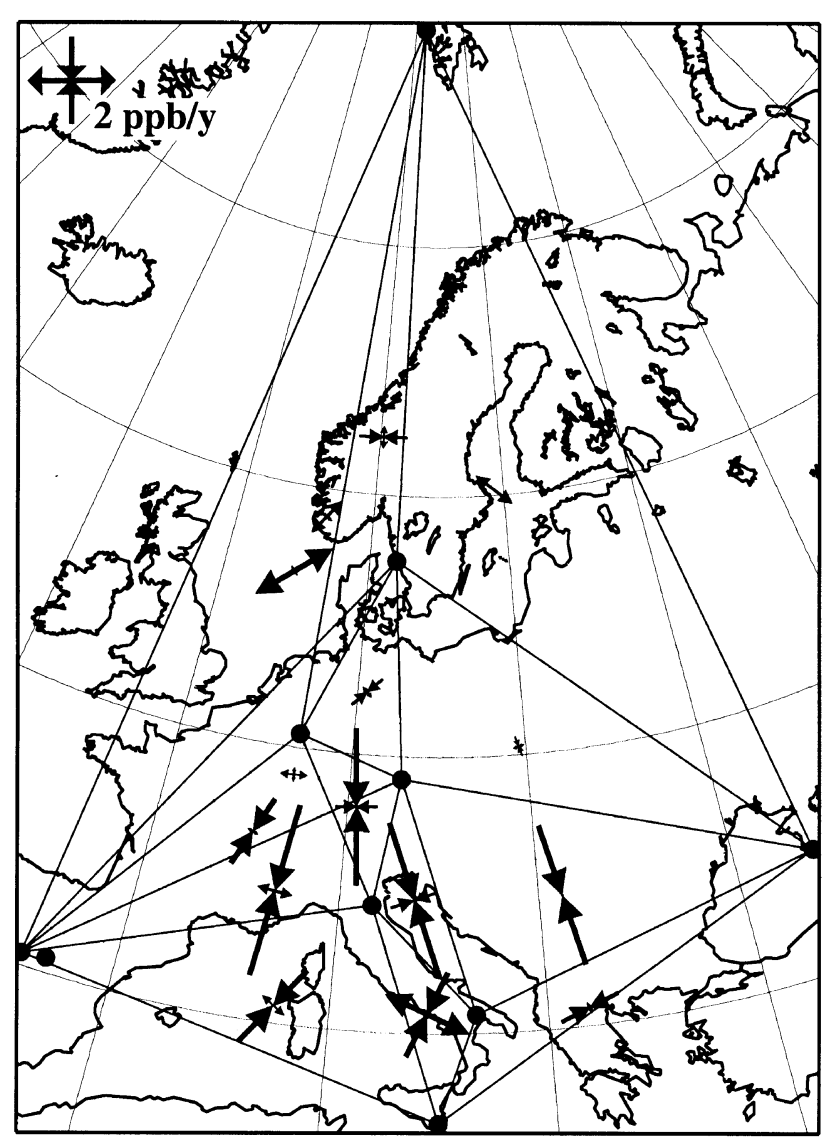

Fig. 5. Results for strain rates in Europe on a large scale.
In our computations we assumed zero vertical motion at Wettzell and all vertical results given are relative to Wettzell. Any real vertical motion at Wettzell which cannot be excluded has to be taken into account if one wants to derive absolute vertical changes. We do not expect it to be any larger than $0.5 \mathrm{~mm} / \mathrm{yr}$ from local surveying (Schlüter, pers. communication). The predicted subsidence of Wettzell based on the post-glacial rebound model used before is $0.12 \mathrm{~mm} / \mathrm{yr}$ and this value has for the sake of consistency been accounted for in the comparisons for Ny Ålesund and Onsala given before.

\subsection{Strain rate analysis}

The observed topocentric station movements were used in a further step to perform a strain rate analysis in the network (Altiner, 1996). For this purpose the network was split up into finite triangle elements each constructed by three nearby stations. The metric tensor of the geographical ellipsoidal coordinates was used and the complete surface deformation tensor of each finite element calculated. After transformation into a local Cartesian coordinate system on the reference surface the components of the strain rate tensor can be displayed graphically (see Fig. 5). The derived strain rates do not exceed $5 \mathrm{ppb} / \mathrm{yr}$. The largest strain rates are detected in the triangles that include the stations Wettzell and Medicina. The two triangles EffelsbergWettzell-Medicina and Wettzell-Medicina-Matera show only compressional strain rates, the triangles Simeiz-Ny ÅlesundOnsala and Ny Ålesund-Wettzell-Simeiz only extensional strain rates, while all other triangles show both type of strain rates. In the northeastern part of the network the strain rates are extremely small.

\section{Conclusions}

Operating now for nearly 10 years the European Geodetic VLBI Network has matured to a reliable high accuracy geodetic and geodynamic observing network. With funding from the EU the first stage of research concentrating on tectonically induced horizontal station motion has been completed producing remarkable results. Geoscientists may use these results to infer upper bounds on present-day tectonics in the areas of interest. In addition, precise station coordinates and reliable time series are available from these measurements. Although GPS measurements using permanent stations also provide very accurate results for global and regional networks, the European VLBI network has the benefit of being inertially tied and having a much longer time series of data. In this respect the VLBI network may serve as a precise reference for the densification of regional and local networks using GPS observations. Synergy effects will arise from combinations of VLBI and the much denser spaced GPS networks for the purpose of strain rate analysis in Europe.

At present, the observations in the network and the investigations in the data gathered are focused on the vertical components. The results are very promising but the correlation between the vertical components and atmospheric refraction presents an obstacle which still has to be overcome (Campbell, 1997).

On the other hand, the comparably higher noise in the results will be compensated for by a longer time series and allow significant signals to emerge. However, this approach bears the deficit that man-made changes in the heights of the 
stations may not be separated from natural causes. Therefore, the project continues the endeavour to improve the accuracy by optimising equipment, observing strategies, refraction models and analysis methods. This includes the geodetic monitoring of the telescope reference points, application of atmospheric loading effects and corrections due to thermal deformation of the radio telescopes.

For the future we hope to extend and densify the European geodetic VLBI network. In particular a further extension to the east and the north is desirable. This would allow an improved tie to the stable east part of the European plate and an improved determination of post-glacial rebound effects.

Acknowledgments. Rüdiger Haas and Erwan Gueguen are supported by the EU within the TMR programme under contract FMRX-CT960071. Hans-Georg Scherneck is supported by grants from the Natural Science Research Council of Sweden under account G-AA/GU 03590. The indispensable efforts of the staff members of all observatories participating in the series and the staff at the Bonn correlator is gratefully acknowledged. This research has made use of NASA Goddard Space Flight Center's VLBI terrestrial reference solution $1102 \mathrm{~g}$, August 1998. Maps were produced with the GMT software package (Wessel and Smith, 1995). We thank Dr. T. Herring and Dr. H. Schuh for constructive reviews of the manuscript.

\section{References}

Albarello, D., E. Mantovani, D. Babbucci, and C. Tamburelli, Africa-Eurasia kinematics: main constraints and uncertainties, Tectonophysics, 243, 2536, 1995.

Altiner, Y., Geometrische Modellierung innerer und äußerer Deformationen der Erdoberfläche, Deutsche Geodätische Kommission, Reihe C: Dissertationen, 462, 1996.

Campbell, J., Measurement of Vertical Motion in Europe by VLBI-Further Support of the European Geodetic VLBI network by the European Union, Proc. of the 11th Working Meeting on European VLBI for Geodesy and Astrometry, Chalmers Research Report 177, 227-231, Onsala, Sweden, 1996.

Campbell, J., Measurement of Vertical Motion in Europe by VLBI-Status of the EU-TMR Network, Proc. of the 12th Working Meeting on European VLBI for Geodesy and Astrometry, 1-8, Hønefoss, Norway, 1997.

De Mets, C., R. G. Gordon, D. F. Argus, and S. Stein, Effect of recent revision to the geomagnetic reversal time scale on estimates of current plate motions, Geophys. Res. Lett., 21(20), 2191-2194, 1994.

Dewey, J. F., M. L. Helman, E. Turco, D. H. W. Hutton, and S. D. Knott, Kinematics of the western Mediterranean, Alpine Tectonics. Geol. Soc. Sp. Publ., 45, 265-283, 1989.

Doglioni, C., E. Gueguen, F. Sabat, and M. Fernandez, The western Mediterranean extensional basins and the Alpine orogen, Terra Nova, 9, 109-112, 1997.

Eanes, R. J. and S. Bettadpur, The CSR 3.0 global ocean tide model, Center for Space Research, Technical Memorandum, CSR-TM-95-06, 1995.

Gueguen, E., C. Doglioni, and M. Fernandez, On the post-25 Ma geodynamic evolution of the western Mediterranean, Tectonophysics, 298,
259-269, 1998.

Haas, R., Untersuchungen zu Erddeformationsmodellen zur Auswertung von geodätischen VLBI-Messungen, Deutsche Geodätische Kommission, Reihe C: Dissertationen, 466, 1996.

Lieske, J. H., T. Lederle, W. Fricke, and B. Morando, Expressions for the Precession Quantities Based upon the IAU (1976) System of Astronomical Constants, Astron. Astrophys., 58, 1-16, 1977.

Ma, C. and J. W. Ryan, NASA Space Geodesy Program-GSFC Data Analysis-1998, VLBI Geodetic Results 1979-1998, August, 1998, http://lupus.gsfc.nasa.gov/global/glb.html, 1998.

Ma, C., J. M. Sauber, L. J. Bell, T. A. Clark, D. Gordon, and W. E. Himwich, Measurement of horizontal motions in Alaska using very long baseline interferometry, J. Geophys. Res., 95, 21991-22011, 1990.

McCarthy, D. D., (ed.) IERS Conventions (1996), IERS Technical Note 21, Observatoire de Paris, 1996.

Mitrovica, J. X., J. L. Davis, and I. I. Shapiro, A spectral formalism for computing three-dimensional deformations due to surface loads, 2. Presentday glacial isostatic adjustment, J. Geophys. Res., 99, 7075-7101, 1994.

Nesterov, N. and A. Volvach, Simeiz VLBI station, International VLBI Service for Geodesy and Astrometry, Annual Report 1999, NASA/TP1999-209243, 96-100, 1999.

Niell, A. E., Global mapping functions for the atmosphere delay at radio wavelength, J. Geophys. Res., 101, 3227-3246, 1996.

Nothnagel, A., IRIS-S batch Solutions at the Geodetic Institute of the University of Bonn, Proc. of the 9th Working Meeting on European VLBI for Geodesy and Astrometry, 42-48, Bad Neuenahr, Germany, 1993.

Nothnagel, A. and J. Campbell, European Baseline Rate Determinations with VLBI, Proc. of the 9th Working Meeting on European VLBI for Geodesy and Astrometry, 56-59, Bad Neuenahr, Germany, 1993.

Réhault, J.-P., J. Mascle, and G. Boilot, Evolution géodynamique de la Méditerranée depuis l'Oligocéne, Mém. Soc. géol. It., 27, 85-96, 1984.

Scherneck, H.-G., A comprehensive and tentatively complete summary of oceanic effects in space geodetic baseline measurements, Proc. of the 11th Working Meeting on European VLBI for Geodesy and Astrometry, Chalmers Research Report 177, 121-133, Onsala, Sweden, 1996.

Scherneck, H.-G., J. M. Johansson, J. X. Mitrovica, and J. L. Davis, The BIFROST project: GPS determined 3-D displacement rates in Fennoscandia from 800 days of continuous observations in the SWEPOS network, Tectonophysics, 294, 305-321, 1998.

Seidelmann, P. K., 1980 IAU Nutation: The Final report of the IAU Working Group on Nutation, Celest. Mech., 27, 79-106, 1982.

Tamura, Y., A harmonic development of the tide-generating potential, Bulletin d'Information Marées Terrestres, 99, 6813-6855, 1987.

Tomasi, P., F. Mantovani, M. Negusini, A. Orfei, and P. Sarti, Activities and recent results in Geodynamics, Proc. of the 12th Working Meeting on European VLBI for Geodesy and Astrometry, 102-110, Hønefoss, Norway, 1997.

Tushingham, A. M. and W. R. Peltier, Ice-3G: a new global model of Late Peistocene deglaciation based upon geophysical predictions of postglacial relative sea level change, J. Geophys. Res., 96, 4497-4523, 1991.

Wahr, J. M., Body tides on an elliptical, rotating, elastic and oceanless earth, Geophys. J. R. A. S., 64, 677-703, 1981.

Wessel, P. and W. H. F. Smith, New version of the Generic Mapping Tool released, EOS Trans. $A G U, \mathbf{7 6}, 329,1995$.

R. Haas (e-mail: haas@oso.chalmers.se), E. Gueguen, H.-G. Scherneck, A. Nothnagel, and J. Campbell 